\title{
Regularity of spectral pattern and its effects on the perceptual fusion of harmonics
}

\author{
BRIAN ROBERTS and PETER J. BAILEY \\ University of York, York, England
}

\begin{abstract}
A single even harmonic added to an odd-harmonic complex may be judged as perceptually more salient than the odd harmonics themselves (Roberts \& Bregman, 1991). It is proposed that this effect occurs because the even harmonic is inconsistent with the regular spectral pattern formed by the odd-harmonic complex (the base). Therefore, a reduction in the regularity of the base spectrum should reduce the even-odd difference. Spectral regularity was reduced either by removing base components, or by including components in the base that were inconsistent with its original pattern. Subjects listened to (primarily) harmonic complex tones and rated the clarity of one of the harmonics, cued by a preceding pure tone. Both removing components from the base and including extraneous components in the base reduced the even-odd difference. The results suggest that it is easier to segregate a harmonic from a periodic complex tone when it does not form part of the regular pattern of spectral spacing defined by the other harmonics.
\end{abstract}

Roberts and Bregman (1991) measured the relative ease with which a single, low-numbered, even harmonic could be heard out from a complex tone consisting otherwise only of odd harmonics. They reasoned that if harmonic relations were the only factor governing the perceptual fusion of the added component, it should be as well integrated into the complex as its neighbors. However, the single even harmonic could generally be heard out more easily than its odd-harmonic neighbors for fundamental frequencies $(F 0 \mathrm{~s})$ of 100 and $200 \mathrm{~Hz}$. Current conceptions of the harmonic sieve (Duifhuis, Willems, \& Sluyter, 1982; Scheffers, 1983), or time-domain equivalents based on subharmonic summation (Hermes, 1988), would not predict a special status for the added even harmonic.

None of the principles of auditory grouping previously explored in the literature seemed able to account for Roberts and Bregman's (1991) finding. However, these authors noted that a series of odd harmonics form a regular spectral pattern across frequency, which is locally disrupted by the addition of an even harmonic. Therefore, they suggested that the odd-one-out status of the even harmonic might provide a basis for its segregation from the rest of the complex. They proposed that the effects of spectral regularity were explicable in the time domain, as a result of interactions between partials not fully resolved by the auditory system. Support for this view came from the finding that the "even-odd difference" seen at low $F 0$ s was abolished when the $F 0$ of the

This research was supported by Project Grant 8912658 from the Medical Research Council (U.K.). Our thanks go to Bob Carlyon, Diek Duifhuis, Stephen Handel, and Brian Moore for their valuable comments and criticism. Correspondence concerning this article should be addressed to B. Roberts, School of Psychology, University of Birmingham, Edgbaston, Birmingham B15 2TT, England (e-mail: b.roberts@ bham.ac.uk). complex was increased to $400 \mathrm{~Hz}$. Moreover, Duifhuis (1970, 1971), Hartmann (1988), and Hartmann, McAdams, and Smith (1990) had previously shown that temporal factors can influence the ability of listeners to hear out a component from a complex tone.

Roberts and Bregman (1991) proposed that the greater perceptual salience of the added even harmonic at low $F 0$ s could be explained in terms of a cross-channel comparison of amplitude modulation (AM) rates at the outputs of the auditory filters. Specifically, the addition of a single even harmonic to an odd-harmonic complex results in a local region of the spectrum where the intermodulation rate is half that found elsewhere, which might provide a cue for the segregation of the added component. Roberts and Bailey (1993a) used two experiments to evaluate this temporal-pattern hypothesis. First, the relative phases of the partials were changed to minimize the depth of AM, and second, noise was added to the stimuli to disrupt the regular pattern of AM evoked by the interactions of the odd harmonics. Both manipulations had quite small effects on the even-odd difference, contrary to the predictions of the temporal-pattern hypothesis. Furthermore, the robustness of the even-odd difference in the presence of significant levels of noise suggests that the results were not greatly influenced by distortion products that might be generated by interactions between the added even harmonic and its odd neighbors.

Roberts and Bailey (1993b) have since explored some other possible accounts of the even-odd difference. In particular, they found that the magnitude of the even-odd difference was changed little when one of the neighboring odd harmonics was removed, suggesting that the immediate neighbors were not crucial for the effect. They also found evidence to suggest that the relative ease with which an added even harmonic could be segregated from an odd-harmonic complex was dependent on its imme- 
diate perceptual salience rather than on auditory learning. These findings, together with those of Roberts and Bailey (1993a), have not provided support for an account of the even-odd difference in terms of known temporal mechanisms operating in the peripheral auditory system. ${ }^{1}$ Therefore, an account based on a central pattern recognizer merits consideration. We note that central pattern recognition is a feature of some successful models of the perception of low pitch (see, e.g., Gerson \& Goldstein, 1978).

The findings to date are all consistent with the proposal that it is easier to segregate a harmonic from a periodic complex tone when it does not form part of the regular pattern of spectral spacing defined by the other harmonics. However, it remains unclear to what extent, if at all, regularity of spectral pattern per se plays a key role in this kind of auditory grouping. To establish this, it must be shown that the pattern properties of the partials comprising the spectrum of the odd-harmonic "base" directly affect the magnitude of the even-odd difference. Therefore, in the experiments reported here we attempted to manipulate the spectral properties that might specify the components of the base as constituting a coherent entity to which the added component does not belong. We have not attempted to define "pattern" or "regularity" in a quantitative manner, but have based our approach on the idea that each member of a regular series acts as a predictor of other members of that series. Specifically, those spectral components that are highly predictable from the spectral location of others should tend to be grouped together and those that are not should tend to be segregated.

The most direct way of reducing the predictability of the frequency relations between the partials of an oddharmonic complex is to reduce the number of partials that make up the complex. Evidence that this strategy might be effective comes from a comparison of the studies of Bregman and Pinker (1978) and Bregman and Doehring (1984). Some of the stimuli used in the latter experiment consisted of a repeating cycle of a pure tone and a complex of three partials. For these stimuli, the frequency of the pure tone matched that of the middle partial in the complex, so that in some instances it captured the middle partial into a sequential stream. In some cases, the three partials were spaced by octaves in the ratio $4: 2: 1$. In others, the middle partial was mistuned downward by a quarter octave. Bregman and Doehring found that it was much easier to capture the middle partial when it was mistuned relative to the others. In contrast, Bregman and Pinker found no evidence that harmonic relations affected segregation when a two-partial complex was used in an otherwise similar context.

Bregman (1990) suggested that the outer two partials of the three-partial complex defined a harmonic frame into which the middle partial did not fit when it was mistuned from an octave ratio. He argued that the effects of harmonic relations were not apparent in the Bregman and Pinker (1978) study because it is not sufficient to have just a single partial define a potential harmonic series into which the other does, or does not, fit. On this basis, one might expect a decrease in the number of partials that maintain a harmonic relation to one another to reduce the perceptual isolation of a partial that is mistuned from a harmonic value.

\section{EXPERIMENT 1}

By analogy with Bregman's (1990) reasoning, a reduction in the number of components making up the odd-harmonic base might be expected to reduce the oddone-out status of the single, added even harmonic. This is because there would be fewer components contributing to a well-defined pattern against which a local inconsistency might be detected. There is also the question of whether or not some components of the base are more important than others for the occurrence of an even-odd difference. In the first experiment, the number of components in the base was reduced from that used in the earlier studies of Roberts and his colleagues (Roberts \& Bailey, 1993a, 1993b; Roberts \& Bregman, 1991). We employed the strategy of removing progressively from the base the components that constituted the upper and lower edges of the spectrum. The intention was to investigate both the importance of the total number of base components and the relative importance of the lower and the higher harmonics in the base.

\section{Method}

Subjects. Ten subjects participated, all of whom reported normal hearing. They were drawn, according to their availability, from a panel of listeners who had successfully completed a screening procedure. ${ }^{2}$ All 10 subjects had taken part in at least one previous study by the authors.

Stimuli and Conditions. Each stimulus was a sequence comprising a pure tone and a complex tone separated by a silent interval of $50 \mathrm{msec}$. The $F 0$ of the complex tone was $100 \mathrm{~Hz}$. The standard form of the complex tone (the base) consisted only of odd harmonics of $F 0$, the particular set of components varying with condition (see below). For each condition, two further variants of the complex were derived from the base by adding a single even harmonic of $F 0$, either 6 or 8 . The frequency of the pure tone was chosen to match that of one of the harmonics 5-9, present in the complex tone. When the complex tone consisted of the base plus an even harmonic, the pure tone always matched the frequency of the even harmonic.

Experiment 1 was subdivided into seven conditions. These differed from one another only in the particular subset of odd harmonics that made up the base. In Condition 1, the base consisted of all odd harmonics of $F 0$, up to and including harmonic 15 . This condition served as a reference, because the spectrum of the base was the same as that employed in several previous experiments (Roberts \& Bailey, 1993a, 1993b). There was no discernible change in the low pitch of the complex resulting from the addition of an even harmonic to the base. The base used in the other conditions differed from Condition 1 in which of its components were removed. Harmonic 15 was not present in any of these conditions. ${ }^{3}$ This ensured that there was an equal number of odd harmonics on each side of the set of harmonics tested, 5-9, that could be removed. These were harmonics 1 and 3 on the low side of the test set, and harmonics 11 and 13 on the high side. Harmonics 5, 7, and 
9 were always present. The odd-numbered harmonics that made up the base for Conditions 2-6 were $1-11,1-9,3-13,5-13$, and 3-11, respectively. The base for Condition 7 consisted only of the "core" set of odd harmonics: 5,7 , and 9.

All stimuli were generated with MITSYN software (see Henke, 1987). For each stimulus, the complex tone consisted of equalamplitude ( $63 \mathrm{~dB} \mathrm{SPL}$ ) components, except for the $F 0$ component (when present), which was boosted by $6 \mathrm{~dB}$ to counteract the effects of decreased auditory sensitivity at low frequencies. Therefore, the overall level of the odd-harmonic base employed in Condition 1 was about $74 \mathrm{~dB}$ SPL. The component levels of the other bases were equal to those of their counterparts in Condition 1. The pure tone was presented at the same level as the corresponding harmonic in the complex tone. All components were in cosine phase. Both the pure tone and the complex tone were presented for a duration of $440 \mathrm{msec}$, including linear onset and offset ramps of $20 \mathrm{msec}$ each. Stimuli were synthesized at a sampling rate of $10 \mathrm{kHz}$ and played back via a 16-bit D/A converter. They were lowpass filtered with a corner frequency of $3.5 \mathrm{kHz}$ (linear phase, 100-dB/oct slope) and were presented binaurally over Sennheiser HD414 SL earphones. The levels of the stimuli were set with a programmable attenuator and were calibrated with a sound-level meter (Brïlel \& Kjaer Type 2203, linear weighting) connected to the earphones by an artificial ear (Type 4153).

Task. A clarity rating procedure was used, in which subjects were required to listen for a cued harmonic in a complex tone and to judge its perceived clarity. The procedure was designed to measure the immediate perceptual salience of the cued harmonic, rather than the accuracy with which that harmonic could be resolved from the complex. ${ }^{4}$

Subjects were told that on each trial they would hear a sequence of two tones, separated by a brief silent interval. The first tone was described as "pure" in quality, and the second, as "rich" in quality. A copy of the pure tone was said to be embedded in the rich tone. The subjects' task was to listen carefully to the pure tone and to rate how clearly they could hear its repetition in the rich tone. They were asked to ignore the "background" formed by the rich tone as much as possible when listening for the sequence comprising the pure tone and its "copy" in the rich tone. They were also asked to ignore any other salient perceptual qualities that might be associated with the rich tone. Subjects were not provided with a definition of clarity in terms of specific auditory qualities. Ratings of perceived clarity were made on a keyboard, using a scale of 1-7. The extremes of the scale ( 1 and 7) indicated least clear and most clear repetitions, respectively. Subjects were encouraged to use the full range of responses available to them. They were allowed to listen to each stimulus only once before choosing their response, and they received no feedback of any kind. After each response, there was an interval of about $1 \mathrm{sec}$ before the next trial. An identical task was used in the previous studies of Roberts and Bailey (1993a, 1993b).

Procedure. The stimuli were presented as 10 blocks of trials in the main session. Each block consisted of a single presentation of the five stimuli corresponding to harmonic numbers 5-9 for each of the seven conditions. The order of presentation within a block was randomized anew for every repetition of that block. The total number of stimuli presented to subjects during this session was 350 ( 7 conditions $\times 5$ harmonics $\times 10$ repetitions). Subjects were free to rest at any time.

Immediately before the main session, subjects were given a short practice run intended to help anchor their judgments on the response scale. First, subjects were given one block of each of the 35 stimuli. At this stage, they were allowed to hear a given stimulus as many times as they wished before choosing a response. Second, they were given another two blocks of stimuli. At this stage, as in the main session, subjects were allowed to listen to each stimulus only once before responding. In the main session, each subject made a total of 10 responses per stimulus, and the mean of these 10 responses was taken as the clarity score for that stimulus. These clarity scores were then averaged across the 10 subjects to give a mean clarity score for each stimulus.

\section{Results}

The results are displayed in three figures. Figure 1 shows the effects of removing the higher components from the base: panels a-c correspond to Conditions 1,2 , and 3 . Figure 2 shows the effects of removing the lower components from the base: panels a-c correspond to Conditions 1, 4, and 5. Figure 3 shows the effects of removing components from the base on both sides of the test set: panels a-c correspond to Conditions 1,6 , and 7 . For these figures, each panel shows the mean clarity scores, with standard errors, for harmonic numbers 5-9. Schematic spectra indicating the components that make up the base are included. These spectra are not to scale with the abscissa.

To assess the significance of changes in the scores across harmonic number, a separate analysis of variance was performed for each condition. A planned comparison of the scores for the odd and even harmonic numbers was achieved by using a contrast within the harmonic number factor. A mean even-odd difference score was obtained for each condition by subtracting the overall mean score for the odd harmonics tested from the overall mean score for the even harmonics tested. The results are considered separately for the three sets of conditions corresponding to Figures 1-3.

The mean even-odd difference score (with standard error) for Condition 1 was $0.89(.28)$. This was the largest difference score for the seven conditions. For Condition 1 , a reliable effect of harmonic number on clarity was found $[F(4,36)=5.93, p<.001]$, and a planned comparison between the odd and the even harmonics tested showed that the difference in clarity between these two sets of components was also reliable $[F(1,9)=10.13$, $p=.011]$. This indicates that the single even harmonic could generally be heard out more clearly than its odd neighbors in Condition 1, suggesting that the integration of the added even harmonic into the complex tone was weaker than that of the odd harmonics. ${ }^{5}$ The result for this reference condition is consistent with previous findings (Roberts \& Bailey, 1993a, 1993b; Roberts \& Bregman, 1991).

The mean even-odd difference scores (with standard errors) for Conditions 2 and 3 were 0.54 (.334) and 0.38 (.22), respectively. In contrast with Condition 1 , a reliable effect of harmonic number on clarity was not found for either of these conditions [Condition $2, F(4,36)=$ $1.44, p=.242$; Condition $3, F(4,36)=1.42, p=.246]$. Furthermore, a planned comparison between the odd and the even harmonics tested showed no reliable difference in clarity between these two sets of components in either condition [Condition 2, $F(1,9)=2.58, p=.143$; Condition $3, F(1,9)=2.91, p=.122]$. These results indicate that removing the higher components from the base reduced or abolished the even-odd difference for the test set of harmonics. Figure 1 shows that this primarily re- 


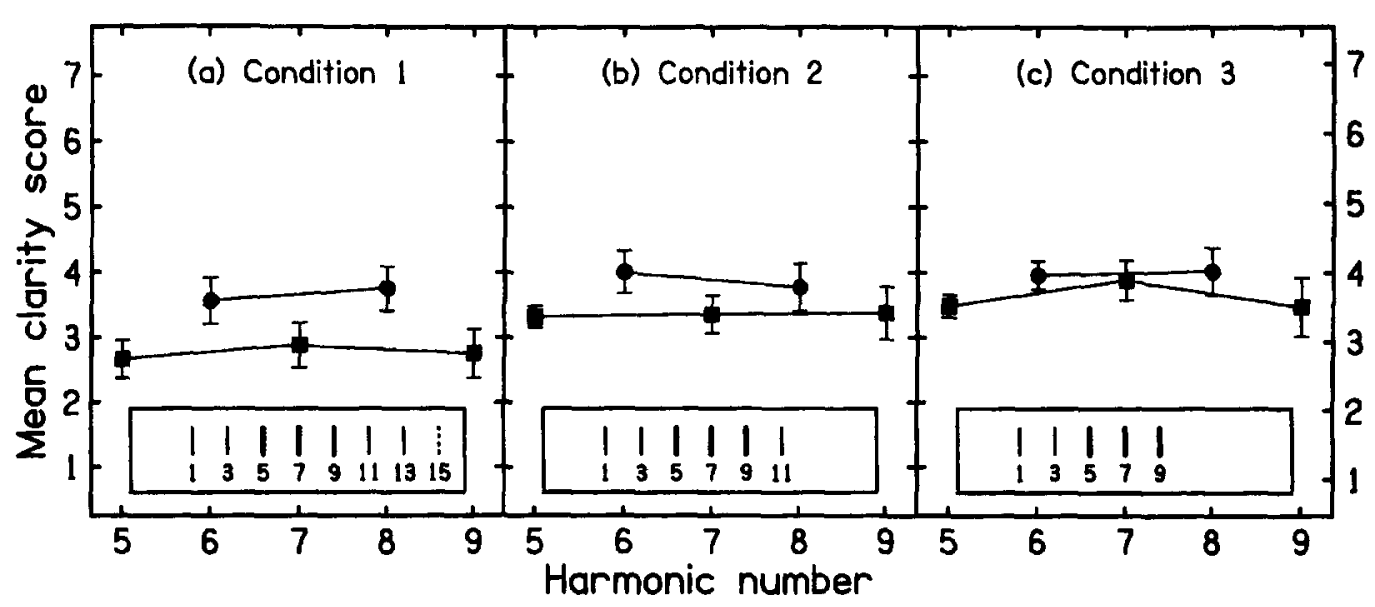

Figure 1. Panels a-c correspond to Conditions 1, 2, and 3, respectively. Each panel shows the mean clarity scores (with standard errors computed from the means for the 10 subjects) across harmonic numbers 5-9. The mean scores for odd and even harmonic numbers are represented by filled squares and filled circles, respectively. The scores were derived from ratings of perceived clarity (see text). The minimum score possible was 1 , and the maximum score possible was 7 . Each panel includes a schematic spectrum to show the odd harmonics that made up the base. These spectra are not to scale with the horizontal axis, but individual harmonic numbers are given. The core set of harmonics $(5,7$, and 9$)$ is depicted by bold lines. Harmonic 15, which is present only in Condition 1, is depicted by a dashed line.

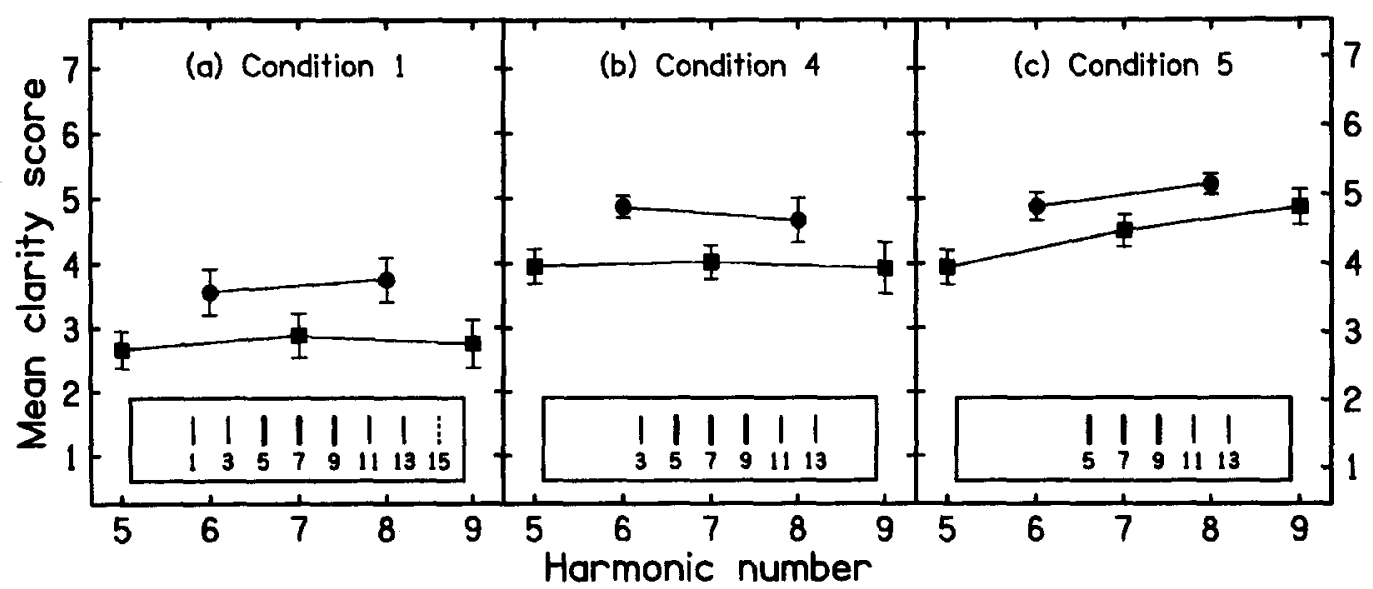

Figure 2. Panels a-c correspond to Conditions 1, 4, and 5, respectively. Each panel shows the mean clarity scores (with standard errors computed from the means for the 10 subjects) across harmonic numbers $5-9$. The mean scores for odd and even harmonic numbers are represented by filled squares and filled circles, respectively. The scores were derived from ratings of perceived clarity (see text). The minimum score possible was 1 , and the maximum score possible was 7 . Each panel includes a schematic spectrum to show the odd harmonics that made up the base. These spectra are not to scale with the horizontal axis, but individual harmonic numbers are given. The core set of harmonics $(5,7$, and 9$)$ is depicted by bold lines. Harmonic 15, which is present only in Condition 1, is depicted by a dashed line.

flects a rise in the clarity of the odd harmonics rather than a fall in the clarity of the evens.

The mean even-odd difference scores (with standard errors) for Conditions 4 and 5 were 0.79 (.28) and 0.61 (.21), respectively. As for Condition 1, a reliable effect of harmonic number on clarity was found for both of these conditions [Condition $4, F(4,36)=3.08, p=.028$; Condition $5, F(4,36)=6.96, p<.001]$. Furthermore, a planned comparison between the odd and the even harmonics tested showed that the difference in clarity between these two sets of components was also reliable for both conditions [Condition $4, F(1,9)=8.24, p=.018$; Condition $5, F(1,9)=8.73, p=.016]$. These results indicate that removing the lower components from the base had less effect on the even-odd difference for the test set of harmonics than did the removal of the higher components. However, the removal of the lower components, particularly the $F 0$ component, leads to a substantial increase in the clarity scores for all the harmonics tested (see Figure 2). For Conditions 4 and 5, there is an increase in the overall mean clarity scores of 1.16 and 1.56 , respectively, relative to the scores in Condition 1. 


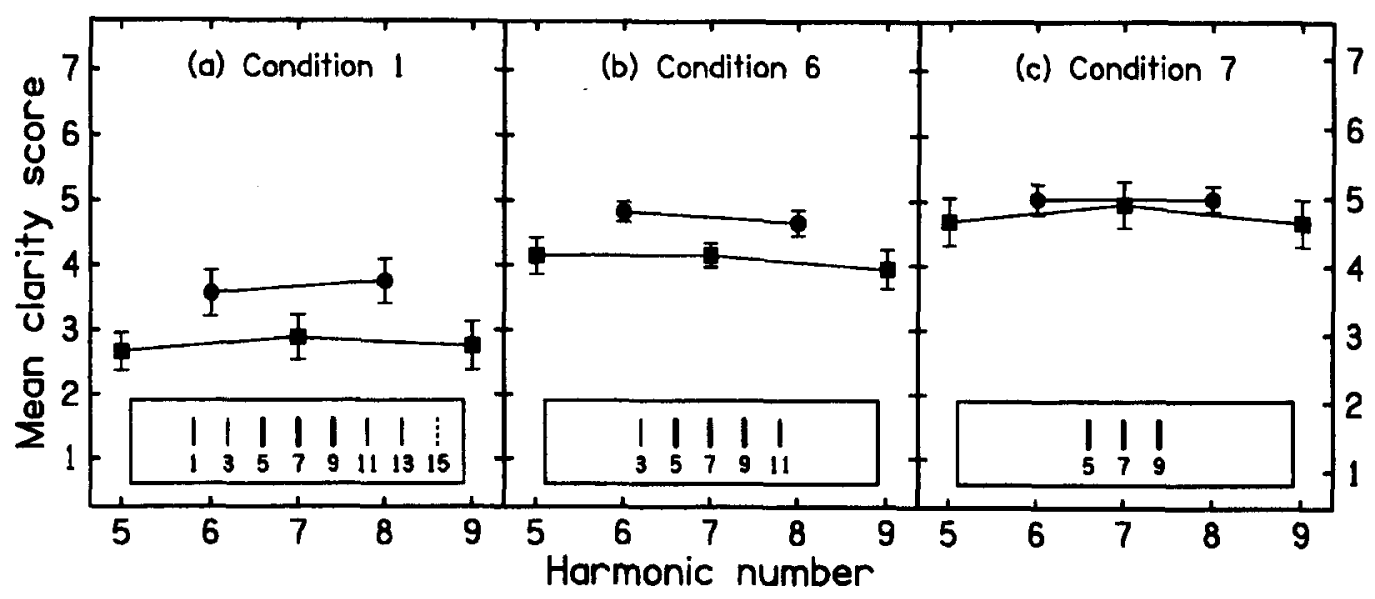

Figure 3. Panels a-c correspond to Conditions 1,6, and 7, respectively. Each panel shows the mean clarity scores (with standard errors computed from the means for the 10 subjects) across harmonic numbers 5-9. The mean scores for odd and even harmonic numbers are represented by filled squares and filled circles, respectively. The scores were derived from ratings of perceived clarity (see text). The minimum score possible was 1 , and the maximum score possible was 7 . Each panel includes a schematic spectrum to show the odd harmonies that made up the base. These spectra are not to scale with the horizontal axis, but individual harmonic numbers are given. The core set of harmonics $(5,7$, and 9$)$ is depicted by bold lines. Harmonic 15, which is present only in Condition 1, is depicted by a dashed line.

The mean even-odd difference scores (with standard errors) for Conditions 6 and 7 were $0.66(.22)$ and 0.27 (.21), respectively. A reliable effect of harmonic number on clarity was found for Condition $6[F(4,36)=4.06$, $p=.008]$, but not for Condition $7[F(4,36)=0.71, p>$ $.500]$. Furthermore, a planned comparison between the odd and the even harmonics tested showed that the difference in clarity between these two sets of components was reliable for Condition $6[F(1,9)=9.07, p=.015]$, but not for Condition $7[F(1,9)=1.68, p=.227]$. These results indicate that removing both the higher and lower components from the base reduced and finally abolished the even-odd difference for the test set of harmonics. ${ }^{6}$ Figure 3 shows that there was also a general increase in clarity scores across conditions, suggesting that removing base components both lower and higher in harmonic number than the test set combines the effects of removing either set alone (see Figures 1 and 2).

\section{Discussion}

The principal finding is that a reduction in the number of base components can reduce or abolish the greater tendency for the added even harmonic to segregate from the odd-harmonic complex. The even-odd difference found when the base consists of the odd harmonics 1-15 decreases both in magnitude and reliability as the number of components constituting the base is reduced. The greatest reduction in the even-odd difference is seen when the higher odd harmonics are removed from the base. When the base consists of only three odd harmonics, 5, 7, and 9, the even-odd difference is small and unreliable. One might argue from Figure 3 that this merely reflects a ceiling effect on subjects' judgments. However, Figure 1 shows that removing the higher components from the base can reduce the even-odd difference when the clarity scores for the even harmonics are well below their peak value.

This experiment has also demonstrated a general increase in clarity scores for both odd and even harmonic numbers when the lower components are removed from the base, indicating a general increase in the tendency for all the components to be heard out from the complex. This may reflect a reduction in the perceptual coherence of the odd-harmonic complex, particularly as the rise in clarity is most marked when the $F 0$ component is removed (Condition 4). ${ }^{7}$ Overall, however, these results support the hypothesis that it is the status of the added even harmonic as the odd-one-out that underlies the even-odd difference, and they suggest that spectral regularity can act as an important factor in auditory grouping. The components that do not form part of the test set of harmonics may help define a regular spectral pattern for the base with which the added even harmonic is inconsistent.

\section{EXPERIMENT 2}

Experiment 1 showed that the even-odd difference score is reduced more by the removal of some components from the odd-harmonic base than by the removal of others. Clearly, it is not simply the number of base components that matters, but also their distribution across frequency. However, we have not demonstrated conclusively that the even-odd difference depends on the pattern properties of the base spectrum. It is still possible that the important factor is the presence of energy in particular frequency regions, rather than the precise frequency relations between the base components. If the key factor is the spectral regularity of the base, it should also be possible to reduce the even-odd difference with- 
out reducing the number of base components. This might be achieved by the inclusion of extra components in the base that are inconsistent with the spectral pattern formed by the odd harmonics. The resulting change in the base pattern should reduce the odd-one-out status of the added even harmonic, making it less likely to segregate from the complex.

In Experiment 2, we chose to include one or more even harmonics in the base, since their inclusion should begin to change the spectral pattern of the base toward one with which the added even harmonic was more consistent. The extra even harmonics had to be chosen from outside the test set of harmonics (1-9 here), so higher even harmonics were used (10 and above). The question addressed was whether the inclusion of higher even harmonics in the base could reduce the greater ease with which an added, low-numbered, even harmonic would be heard out from the complex, relative to its odd neighbors.

\section{Method}

Subjects and Design. Ten subjects from the panel of listeners took part, of whom 6 were also participants in Experiment 1. Only 2 subjects had not taken part in any previous experiments by the authors. Four conditions were employed. The total number of tri- als in the main session was 360 (4 conditions $\times 9$ harmonics $\times 10$ repetitions).

Stimuli and Conditions. Four variants of the complex tone were derived from the base in each condition by adding a single even harmonic of $F 0: 2,4,6$, or 8 . The frequency of the pure tone was chosen to match that of one of the harmonics $1-9$, present in the complex tone. The set of components making up the base varied with condition. In Condition 1 , the base consisted of all odd harmonics of $F 0$, up to and including harmonic 15 . This served as a reference condition. In the other three conditions, the base contained one or more extra components. These extra components were higher numbered even harmonics, chosen to fall in the region immediately above the test set of harmonics (1-9). For Conditions $2-4$, respectively, the base included harmonic 10 , harmonics 10 and 12 , and harmonics 10,12 , and 14 .

\section{Results}

The results are shown in Figure 4. Panels a-d correspond to Conditions 1-4, respectively. Each panel shows the mean clarity scores, with standard errors, for harmonic numbers $1-9$.

A reliable effect of harmonic number on clarity was found for all four conditions [Condition $1, F(8,72)=8.23$, $p<.001$; Condition $2, F(8,72)=4.58, p<.001$; Condition $3, F(8,72)=4.19, p<.001 ;$ Condition $4, F(8,72)=$

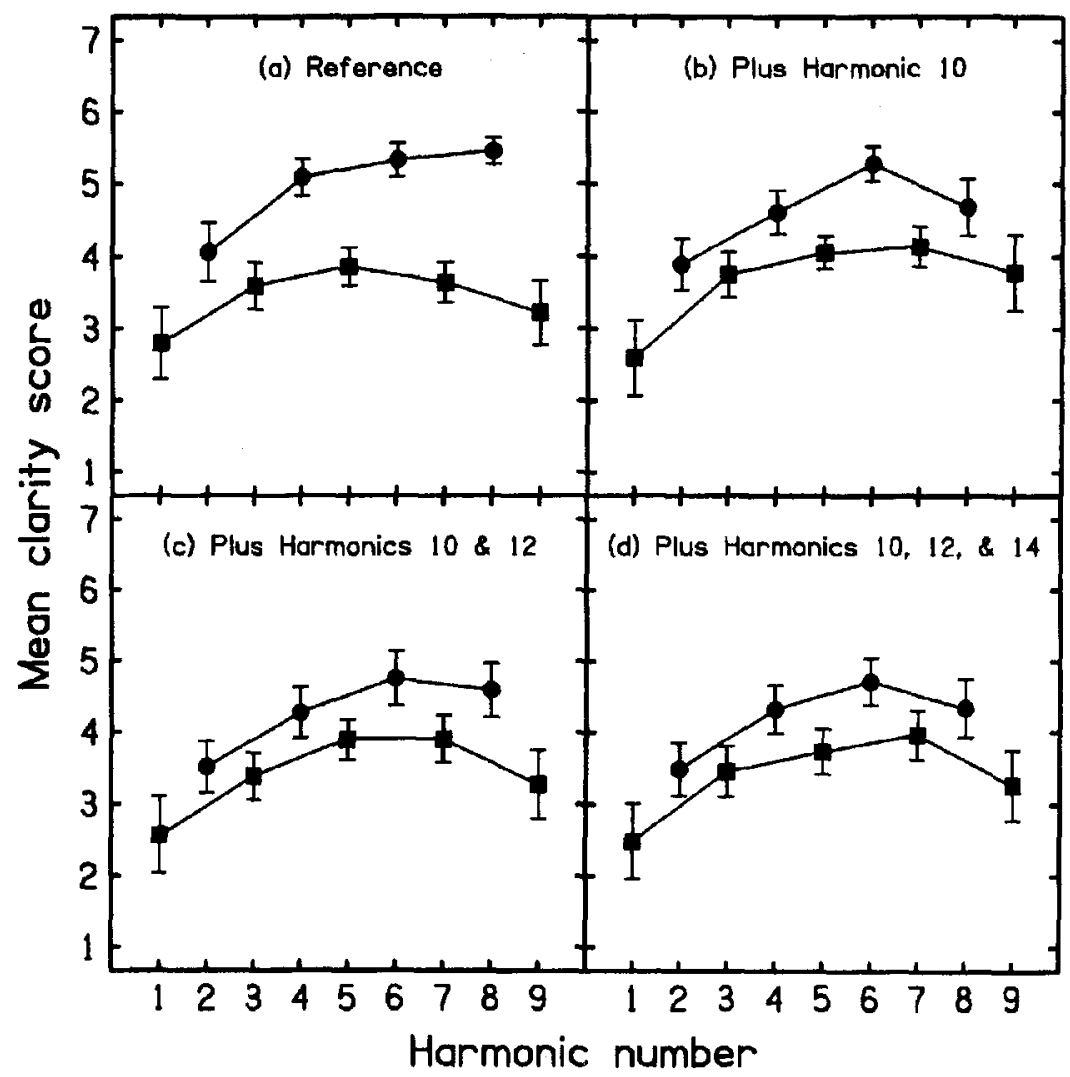

Figure 4. Panels a-d correspond to Conditions 1-4, respectively. Each panel shows the mean clarity scores (with standard errors computed from the means for the 10 subjects) across harmonic numbers 1-9. The mean scores for odd and even harmonic numbers are represented by filled squares and filled circles, respectively. The scores were derived from ratings of perceived clarity (see text). The minimum score possible was 1 and the maximum score possible was 7 . 
$4.07, p<.001]$. Moreover, the planned comparison between the odd and the even harmonics showed that the difference in clarity between these two sets of components was reliable in all cases [Condition $1, F(1,9)=90.21$, $p<.001$; Condition $2, F(1,9)=39.08, p<.001$; Condition $3, F(1,9)=36.63, p<.001$; Condition $4, F(1,9)=$ $38.18, p<.001]$. These results indicate that an even harmonic from the test set could generally be heard out more clearly than its odd neighbors in all four conditions. This shows that the inclusion of one or more higher even harmonics in the base did not abolish the greater tendency for the added even harmonic to be segregated from the complex.

Although a reliable even-odd difference was found in each condition, the higher even harmonics included in the base were not without effect. The mean even-odd difference scores (with standard errors) for Conditions $1-4$ were $1.56(.17), 0.95(.15), 0.88(.15)$, and 0.84 (.14), respectively. These scores were reliably different $[F(3,27)=16.30, p<.001]$. Pairwise comparisons (Tukey's HSD) showed reliable differences between Condition 1 and all other conditions [Condition 1 vs. $2=$ $0.61, p<.01 ; 1$ vs. $3=0.68, p<.01 ; 1$ vs. $4=0.72, p<$ $.01]$. None of the pairwise comparisons between Conditions 2,3 , and 4 revealed reliable differences. These findings indicate a reliable reduction in the magnitude of the even-odd difference when higher even harmonics are included in the base, with the greatest change resulting from the inclusion of harmonic 10 . The fact that including harmonics 12 and 14 in the base had at best a small effect may reflect their greater spectral distance from the test set.

The source of the reduction in the even-odd difference when higher even harmonics were included in the base can be illustrated most clearly by considering the data for Conditions 2-4 relative to the data for Condition 1. For each subject, a clarity shift was obtained for each harmonic tested in Conditions $2-4$. This was achieved by taking the clarity score for the appropriate stimulus and subtracting from it the clarity score for the corresponding stimulus in Condition 1 . These clarity shifts were then averaged across the 10 subjects to give a mean clarity shift for each stimulus. The resulting data are shown in Figure 5. Panels a-c correspond to Conditions $2-4$, respectively. Each panel shows the mean clarity shifts, with standard errors, for harmonic numbers 1-9.

Figure 5 shows clearly that the inclusion of the higher even harmonics in the base had relatively little effect on the clarity scores for the odd harmonics, with the exception of the higher odd harmonics tested in Condition 2. In Conditions 3 and 4, harmonic 9 was unaffected-even though it was adjacent to the extra base components. Rather, the reduction in the even-odd difference appears to result largely from a reduction in the clarity of the added even harmonic. Figure 5 shows this reduction to be progressive as more higher even harmonics are included in the base. The reduction was especially marked for harmonic 8 , which is the nearest even harmonic in the test set to the extra ones included in the base. This may be because the addition of harmonic 8 to the bases used in Conditions 2-4 completed a local series of five or more successive harmonics.

\section{Discussion}

These data are consistent with the notion that the inclusion of higher even harmonics in the base reduces the odd-one-out status of the added even harmonic. However, a change in the spectral regularity of the base resulting from the inclusion of extra components is not the only plausible explanation of the reduction in the evenodd difference. Two other accounts should also be considered.

First, the inclusion of higher even harmonics in the base reduces the resolution of the adjacent odd harmonics. This may have effects similar to those of reducing the number of odd harmonics in the base, in which case the results of Experiment 2 would be a further demon-

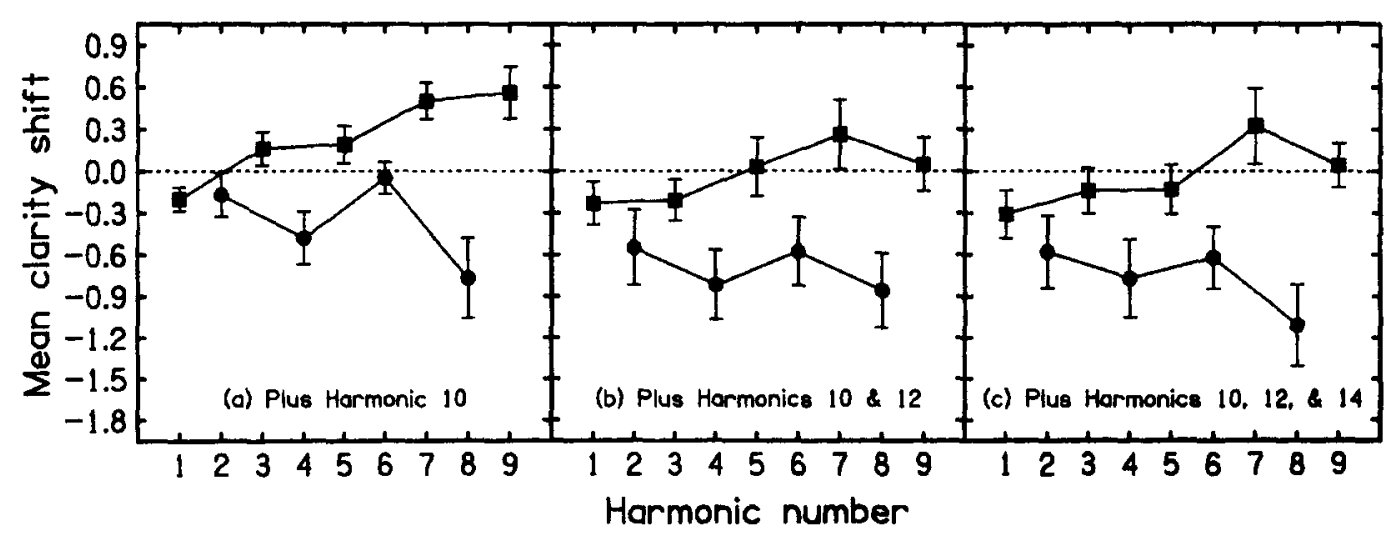

Figure 5. Panels a-c correspond to Conditions 2-4, respectively. Each panel shows the mean clarity shifts (with standard errors computed from the mean shifts for the 10 subjects) across harmonic numbers $1-9$. These scores represent the change in clarity relative to the corresponding harmonic number in Condition 1. Mean clarity shifts for odd and even harmonic numbers are represented by filled squares and filled circles, respectively. 
stration of the effects seen in Experiment 1. This suggestion is difficult to refute directly, because the inclusion of extra components in the base inevitably affects the resolution of the neighboring odd harmonics. ${ }^{8}$ However, this account cannot explain an important difference between Experiments 1 and 2 in the origin of the evenodd difference: The removal of higher odd harmonics from the base acts primarily to increase the clarity of the odd harmonics, whereas the inclusion of higher even harmonics in the base acts primarily to decrease the clarity of the even harmonics. Moreover, the clarities of all the even harmonics tested fell when higher even harmonics were included in the base.

Second, it is possible that the reduction in the evenodd difference found here depended specifically on harmonic relations, since the extra components included in the base were themselves even-numbered harmonics of $F 0$. This account was evaluated directly in a further experiment, designed to explore whether the effects on the even-odd difference found here would generalize to any components that affect the spectral regularity of the base, irrespective of their harmonic status.

\section{EXPERIMENT 3}

In this experiment, we compared the effect on the evenodd difference score of including in the base either har- monic 10 or a component mistuned from a harmonic value, but falling between harmonics 9 and 11. The purpose was to investigate the specificity of the effect of including higher components in the base observed in Experiment 2. If the reduction in the even-odd difference resulted from the reduced regularity of the base relative to the original base spectrum, it should not matter whether or not the extra component employed here was a harmonic of $F 0$.

\section{Method}

Subjects and Design. Ten subjects from the panel of listeners took part, of whom 3 were participants in both Experiments 1 and 2, and a further 4 were participants in Experiment 1 only. Only 1 subject had not taken part in any previous experiments by the authors. Four conditions were employed. The total number of trials in the main session was 360 ( 4 conditions $\times 9$ harmonics $\times 10$ repetitions).

Stimuli and Conditions. As for Experiment 2, the base in Condition 1 consisted of all odd harmonics of $F 0$, up to and including harmonic 15. In the other conditions, the base contained one extra component. This component was chosen to fall between harmonics 9 and 11 , corresponding to frequencies of 900 and $1100 \mathrm{~Hz}$. In Condition 2, the frequency of the extra component was $1000 \mathrm{~Hz}$, the 10th harmonic of $F 0$. In conditions 3 and 4, respectively, the frequency of the extra component was mistuned downward to $950 \mathrm{~Hz}$ and upward to $1050 \mathrm{~Hz}$.

\section{Results}

The results are shown in Figure 6. Panels a-d correspond to Conditions 1-4, respectively. Each panel shows

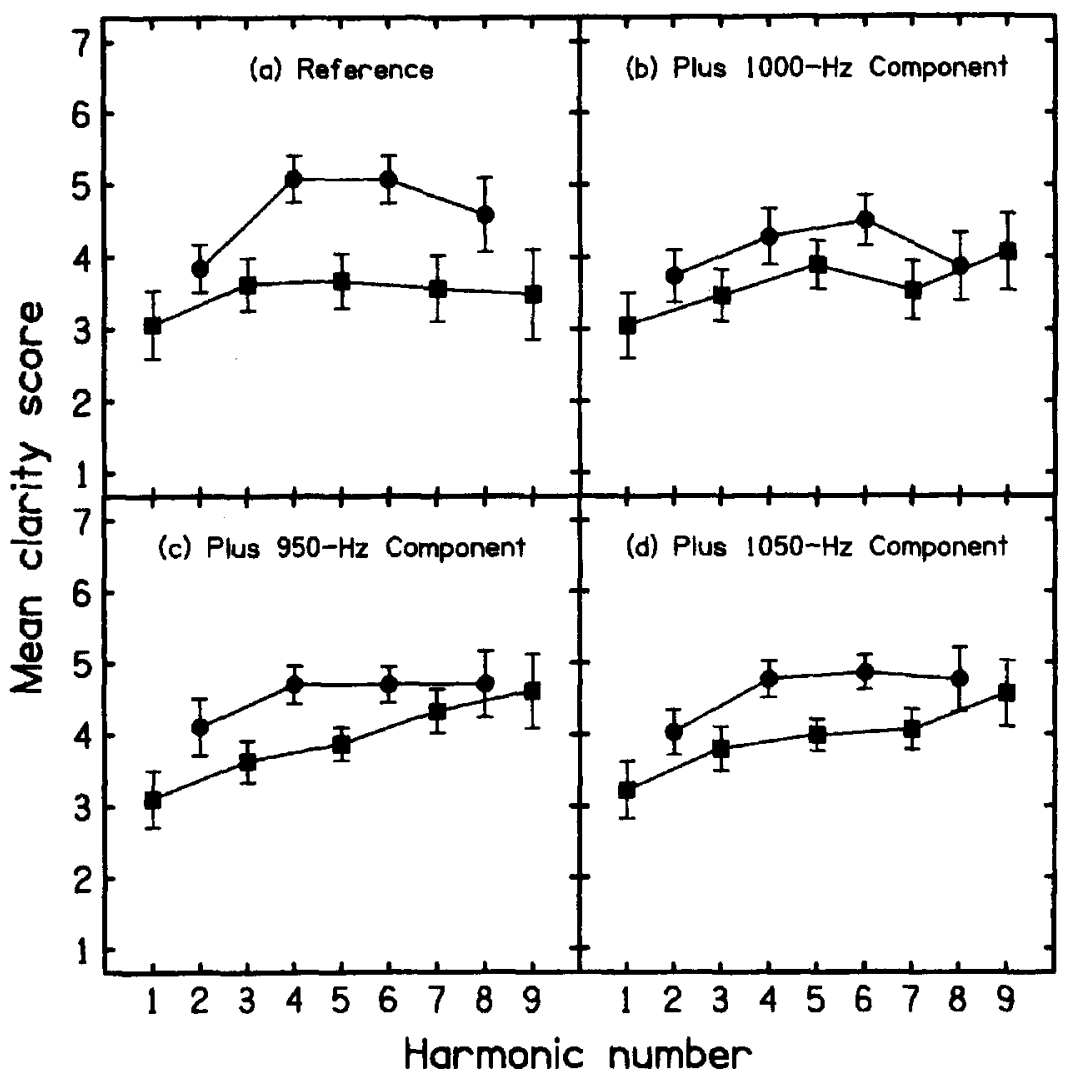

Figure 6. Panels a-d correspond to Conditions 1-4, respectively. Each panel shows the mean clarity scores (with standard errors computed from the means for the 10 subjects) across harmonic numbers 1-9. 
the mean clarity scores, with standard errors, for harmonic numbers $1-9$.

A reliable effect of harmonic number on clarity was found only for Conditions 1,3 , and 4 [Condition $1, F(8,72)$ $=3.59, p=.002$; Condition $2, F(8,72)=1.51, p=.170$; Condition $3, F(8,72)=2.41, p=.023$; Condition 4, $F(8,72)=2.58, p=.015]$. However, the planned comparison between the odd and the even harmonics showed that the difference in clarity between these two sets of components was reliable for all conditions [Condition 1, $F(1,9)=$ 25.38, $p<.001$; Condition 2, $F(1,9)=10.21, p=.011$; Condition $3, F(1,9)=11.73, p=.008$; Condition 4, $F(1,9)=22.11, p=.001]$. These results indicate that the single even harmonic could generally be heard out more clearly than its odd neighbors in all four conditions. This shows that the inclusion of an extra component in the base did not abolish the greater tendency for the added even harmonic to be segregated from the complex.

The mean even-odd difference scores (with standard errors) for Conditions 1-4 were 1.17 (.23), 0.49 (.16), $0.65(.19)$, and $0.68(.14)$, respectively. These scores were reliably different $[F(3,27)=9.57, p<.001]$. Pairwise comparisons (Dunnett's test) showed reliable differences between Condition 1 (control mean) and all other conditions $[1$ vs. $2=0.68, p<.01 ; 1$ vs. $3=0.52$, $p<.01 ; 1$ vs. $4=0.49, p<.01]$. These results indicate a reliable reduction in the magnitude of the even-odd difference when an extra component is included in the base between harmonics 9 and 11 , irrespective of the harmonic status of that component. Although the reduction of the even-odd difference was greatest when the extra component was harmonic 10, a planned comparison of Condition 2 versus Conditions 3 and 4 (pooled) did not reveal a reliable effect $[F(1,9)=4.50, p=.063]$.

Clarity shifts were calculated in the same way as described above for Experiment 2. The resulting data are shown in Figure 7. Panels a-c correspond to Conditions $2-4$, respectively. Each panel shows the mean clarity shifts, with standard errors, for harmonic numbers $1-9$.
The pattern of results in Figure $7 \mathrm{a}$ is broadly similar to that seen in Figure 5a, which represents the results for the equivalent condition in Experiment 2. The inclusion of harmonic 10 in the base reduces the even-odd difference primarily by lowering the clarity of the even harmonics rather than by raising the clarity of the odd harmonics. Conditions 3 and 4 differ from Condition 2 in that the extra component included in the base is mistuned from a harmonic value. The effect of this difference can be seen by comparing Figures $7 \mathrm{~b}$ and $7 \mathrm{c}$ with Figure 7a. Unlike in Condition 2, the clarity of the even harmonics is relatively unchanged by the presence of the extra component in the base in Conditions 3 and 4. Rather, the reduction in the even-odd difference results primarily from a rise in the clarity of the odd harmonics. The extent of this rise in clarity increases progressively across the odd harmonics tested.

\section{Discussion}

This experiment has shown that any extra component included in the base between harmonics 9 and 11 can reduce the even-odd difference for the test set, irrespective of whether or not it is harmonically related to the other components of the base. These data support the idea that the even-odd difference is reduced because the inclusion of any component that is not an odd harmonic of $F 0$ reduces the spectral regularity of the base to which an even harmonic from the test set is added. However, there is some reason to believe that the particular changes in clarity that resulted in a reduced even-odd difference were influenced by the harmonic status of the extra component. This is illustrated by Figure 7 , which shows that the inclusion in the base of harmonic 10 (Condition 2) acts primarily to reduce the clarity of the even harmonics, whereas the inclusion in the base of a mistuned component (Conditions 3 and 4 ) acts primarily to increase the clarity of the odd harmonics.

One possible explanation is that the inclusion of harmonic 10 produces a change in the spectral pattern de-

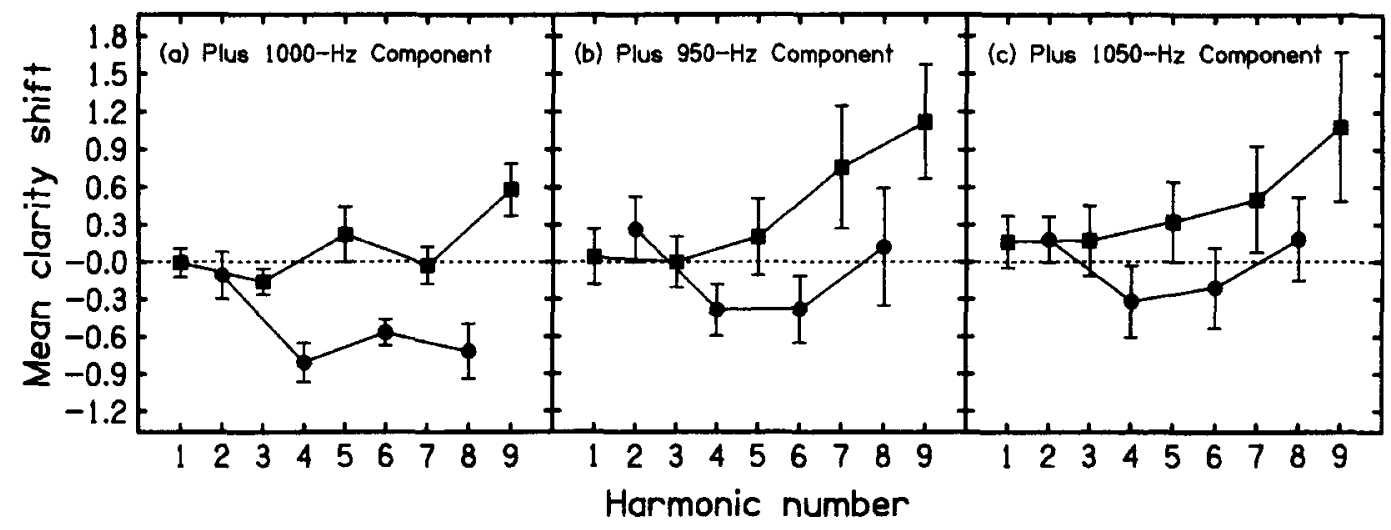

Figure 7. Panels a-c correspond to Conditions 2-4, respectively. Each panel shows the mean clarity shifts (with standard errors computed from the mean shifts for the 10 subjects) across harmonic numbers 1-9. 
fined by the base harmonics that is consistent with that produced by adding an even harmonic from the test set. In contrast, the inclusion of a mistuned component is not predictive of the change in spectral pattern that results from the addition of an even harmonic. Rather, the extra component introduces an element of inharmonicity into the complex. It remains unclear precisely why the inclusion of a mistuned component should result primarily in a rise in the clarity of the odd harmonics, rather than a fall in the clarity of the even harmonics. However, the finding of a general increase in clarity for all the odd harmonics in Conditions 3 and 4, relative to Condition 1 , indicates that a simple account of these data cannot be provided by reference to the consequences of frequency resolution. ${ }^{9}$

\section{GENERAL DISCUSSION}

Roberts and his colleagues have suggested that the integration of a given harmonic into the percept of a complex tone is influenced by how consistent it is with the spectral pattern formed by the other harmonics (Roberts \& Bailey, 1993a, 1993b; Roberts \& Bregman, 1991). We have attempted to evaluate this hypothesis by manipulating the spectral pattern of an odd-harmonic base in ways intended to reduce the hypothesized odd-one-out status of the added even harmonic.

The experiments reported here have shown that the even-odd difference can be reduced either by reducing the number of components in the odd-harmonic base (Experiment 1), or by including extra components in the base that do not fit the original spectral patte rn (Experiment 2). Furthermore, a reduction in the even-odd difference can result from the inclusion in the base of an extra component that is either at or mistuned from a harmonic value (Experiment 3 ). Both removing pattern elements and including elements that change the original pattern might be expected to reduce the odd-one-out status of the added even harmonic.

The overall findings implicate the regularity of the base spectrum as a major factor underlying the even-odd difference. Effects of spectral proximity and of harmonicity have also been evident in the data, but these features appear to be of secondary importance. On the basis of these data, we conclude that the tendency of a single, low-numbered, even harmonic to segregate from an odd-harmonic complex can best be attributed to the local disruption that it causes in the regular pattern of spectral spacing defined by the odd harmonics. Thus far, all the experiments of Roberts and his colleagues have focused on measuring how well harmonic components can be heard out from a complex tone. However, it remains to be established whether or not the proposed effects of spectral regularity are restricted to the perceptual fusion of harmonics. We are addressing this in our continuing research by exploring the effects of spectral pattern on the perceptual organization of inharmonic stimuli (Roberts \& Bailey, in press). This approach may also help elucidate the possible contributions of temporal patterning to these grouping processes.

\section{REFERENCES}

Bregman, A. S. (1990). Auditory scene analysis: The perceptual organization of sound. Cambridge, MA: MIT Press.

Bregman, A. S., \& DoEhring, P. (1984). Fusion of simultaneous tonal glides: The role of parallelness and simple frequency relations. Perception \& Psychophysics, 36, 251-256.

Bregman, A. S., \& Pinker, S. (1978). Auditory streaming and the building of timbre. Canadian Journal of Psychology, 32, 19-31.

DuifHuis, H. (1970). Audibility of high harmonics in a periodic pulse. Journal of the Acoustical Society of America, 48, 888-893.

DuIFHuIs, H. (1971). Audibility of high harmonics in a periodic pulse: II. Time effect. Journal of the Acoustical Society of America, 49, $1155-1162$.

Duifhuis, H., WILlems, L. F., \& Sluyter, R. J. (1982). Measurement of pitch in speech: An implementation of Goldstein's theory of pitch perception. Journal of the Acoustical Society of America, 71, 1568 1580.

Gerson, A., \& Goldstein, J. L. (1978). Evidence for a general template in central optimal processing for pitch of complex tones. Journal of the Acoustical Society of America, 63, 498-510.

Hartmann, W. M. (1988). Pitch perception and the segregation and integration of auditory entities. In G. M. Edelman, W. E. Gall, \& W. M. Cowan (Eds.), Auditory function (pp. 623-645). New York: Wiley.

Hartmann, W. M., McAdams, S., \& Smith, B. K. (1990). Hearing a mistuned harmonic in an otherwise periodic complex tone. Journal of the Acoustical Society of America, 88, 1712-1724.

HENKE, W. L. (1987). MITSYN languages. Belmont, MA: Author.

HeRMEs, D. J. (1988). Measurement of pitch by subharmonic summation. Journal of the Acoustical Society of America, 83, 257-264.

RoBerts, B., \& BaILeY, P. J. (1993a). Spectral pattern and the perceptual fusion of harmonics: I. The role of temporal factors. Journal of the Acoustical Society of America, 94, 3153-3164.

RoBerTS, B., \& BAILEY, P. J. (1993b). Spectral pattern and the perceptual fusion of harmonics: II. A special status for added components? Journal of the Acoustical Society of America, 94, 3165-3177.

ROBERTS, B., \& BAILEY, P. J. (in press). Spectral regularity as a factor distinct from harmonic relations in auditory grouping. Journal of Experimental Psychology: Human Perception \& Performance.

ROBERTS, B., \& BREGMAN, A. S. (1991). Effects of the pattern of spectral spacing on the perceptual fusion of harmonics. Journal of the Acoustical Society of America, 90, 3050-3060.

SCHEFFER, M. T. M. (1983). Simulation of auditory analysis of pitch: An elaboration on the DWS pitch meter. Journal of the Acoustical Society of America, 74, 1716-1725.

Schouten, J. F, Ritsma, R. J., \& Cardozo, B. L. (1962). Pitch of the residue. Journal of the Acoustical Society of America, 34, 14181424 .

\section{NOTES}

1. One of the reviewers has pointed out that the waveforms of oddharmonic complexes are symmetrical around zero, and that this symmetry would be disrupted by the addition of a single even harmonic. Though it remains unclear whether or not the peripheral auditory system is sensitive to such properties of the whole waveform, the relative insensitivity of the even-odd difference to the presence of noise (Roberts \& Bailey, 1993a) does not suggest a key role for changes in waveform symmetry.

2 . In the screening procedure, a clarity rating task was used in selecting the panel of listeners. The stimuli employed were equivalent to those used in Condition 1 of Experiment 2. All 90 stimuli (ten blocks of the nine harmonic numbers tested) were presented, with repeated listening permitted before a response was chosen. Listeners were then required to judge the same set of 90 stimuli again, but this time repeated listening was not permitted. Listeners who, by the end of the 
procedure, reported themselves still unable to hear a "copy" of the pure tone embedded in the complex tone on an appreciable number of trials were excluded from the panel. They amounted to about one fifth of all listeners screened.

3. A pilot study had indicated that the presence or absence of harmonic 15 in the base had no reliable effect on subjects' responses to harmonics in the test set.

4. Roberts and Bailey (1993a) have discussed the design of the clarity procedure in greater detail, and they consider more generally the use of both subjective and performance measures of perceptual segregation.

5. Roberts and Bailey (1993b) noted that auditory learning of the base components could in principle take place, and that this might lead to higher clarity scores for the relatively novel added components. However, they observed that the even-odd difference was not diminished when the $F 0$ of the base was changed across trials as a control for the effects of auditory learning. These authors also demonstrated that the even-odd difference observed with the use of the clarity procedure was not a result of the different spectral context in which the even and odd harmonics were tested. They showed, using a pitch judgment task, that the added even harmonic could often be "captured" into a sequential group by a preceding pure tone that matched the frequency of one of its odd-harmonic neighbors. This shows that the odd neighbors remain more difficult to hear out than the even harmonic when both are tested with the use of the same stimuli.

6. Our working assumption is that the clarity scale is approximately linear over the range that the subjects choose to use. However, a compressive nonlinearity in the use of the scale, for example, would lead to an apparent reduction in the even-odd difference with an increase in mean clarity scores. This could contribute to the pattern of results seen in Figure 3, but it is unlikely to provide a general account of our results. Specifically, the reduction in the even-odd difference across conditions seen in Figure 1 is observed despite relatively constant scores for the even harmonics.

7. Odd-harmonic complexes with few components can also be perceptible as frequency-shifted full complexes (see, e.g., Schouten, Ritsma, \& Cardozo, 1962). Therefore, it is possible that the addition of an even harmonic to such complexes resolves their ambiguity of pitch, and that this effect determines the size of the even-odd difference observed. One might expect this effect to be greatest in Condition 7 , where the odd-harmonic base comprises only harmonics 5,7 , and 9 . However, Condition 7 was the only case for which no even-odd difference was found. This suggests that the effects of pitch were not of primary importance here.

8 . There do not seem to be systematic effects on the clarity scores for harmonic 9 resulting from the inevitable reduction in its resolution when harmonic 10 is also present.

9. The hypothesis that the clarity shifts for harmonic 9 are attributable primarily to the perceptual salience of the extra component, and not to effects on resolution, is supported by the finding that the clarity shifts were not a simple function of the frequency proximity of the extra component in the base, and that they were larger for the mistuned conditions than for the harmonic condition.

(Manuscript received November 7, 1994; revision accepted for publication July 16, 1995.) 